\title{
Continuous \& Piecewise Convex Behavior Of Maximum Values Of Some Generalized Measures Of Fuzzy Cross Entropy.
}

\author{
Author: C.P. Gandhi
}

Associate Professor,Department of Mathematics,

Rayat Bahra Institute of Engineering and Bio-Technology, Kharar,Mohali ,India

Tel +91-9888909197, Fax: 0160-5009680,

E-mail:cchanderr@gmail.com

Abstract-- Consider two fuzzy sets A and B with same supporting points $x_{1}, x_{2}, \ldots \ldots x_{n}$ and the corresponding fuzzy vectors $\left(\mu_{A}\left(x_{1}\right), \mu_{A}\left(x_{2}\right), \ldots \ldots \mu_{A}\left(x_{n}\right)\right)$ and $\left(\mu_{B}\left(x_{1}\right), \mu_{B}\left(x_{2}\right), \ldots \ldots \mu_{B}\left(x_{n}\right)\right)$ respectively, where each $\mu_{A}\left(x_{i}\right), 1 \leq i \leq n$ can vary subject to the total fuzziness $\sum_{i=1}^{n} \mu_{A}\left(x_{i}\right)=k$ and each $\mu_{B}\left(x_{i}\right)$ is known to us. In this paper, a comprehensive and straightforward procedure is made available to find the maximum values of some existing measures of generalized fuzzy cross entropy. Further, the continuous and piecewise convex behavior of the values so obtained is also discussed mathematically as well as graphically. Finally, a numerical example is used to illustrate the proposed method.

Keywords- Fuzzy set, fuzzy vector, fuzzy cross entropy, continuity piecewise convexity, maximum value.

\section{Council for Innovative Research}

Peer Review Research Publishing System

Journal: International Journal of Computers \& Technology

Vol 8, No. 2

editor@cirworld.com

www.cirworld.com, member.cirworld.com 


\section{INTRODUCTION}

n spite of the large diversity of mathematical models that are actually encountered in the domain of entropy optimization problems, Aczel[1] and others endorsed the view that generalized measures of cross-entropy should be introduced whenever the maximization of these measures leads to useful mathematical models in various fields. The optimization of generalized measures fuzzy cross entropy arise very naturally in response to the need to explain the existence of a variety of models in different contexts. As introduced by Zadeh[5].Consider a given fuzzy set $A$ with $n$ supporting points $\left(x_{1}, x_{2}, \ldots \ldots x_{n}\right)$ corresponding to the fuzzy vector $\left(\mu_{A}\left(x_{1}\right), \mu_{A}\left(x_{2}\right), \ldots \ldots \mu_{A}\left(x_{n}\right)\right)$, where $\mu_{A}\left(x_{i}\right)$ is the degree of membership of the elements $x_{i}$ of the set $A$. Our purpose is find the maximum values of some well known measures of generalized fuzzy cross entropy subject to the total fuzziness $\sum_{i=1}^{n} \mu_{A}\left(x_{i}\right)=k, 0 \leq k \leq n$. For this, we have considered some well known generalized measures of fuzzy cross entropy which are given below:

Corresponding to Renyi's [4] measure of fuzzy entropy, Kapur's[]3measure of fuzzy cross entropy is given by the following mathematical expression:

$D_{\alpha}(A: B)=\frac{1}{\alpha-1} \sum_{i=1}^{n} \log \left[\mu_{A}^{\alpha}\left(x_{i}\right) \mu_{B}^{1-\alpha}\left(x_{i}\right)+\left(1-\mu_{A}\left(x_{i}\right)\right)^{\alpha}\left(1-\mu_{B}\left(x_{i}\right)\right)^{1-\alpha}\right], \alpha \neq 1, \alpha>0$

Corresponding to Havrda and Charvet [2] measure of fuzzy entropy,Kapur's[3] measure of fuzzy cross entropy is given by the following mathematical expression:

$$
D^{\alpha}(A: B)=\frac{1}{\alpha-1} \sum_{i=1}^{n}\left[\mu_{A}^{\alpha}\left(x_{i}\right) \mu_{B}^{\alpha-1}\left(x_{i}\right)+\left(1-\mu_{A}\left(x_{i}\right)\right)^{\alpha}\left(1-\mu_{B}\left(x_{i}\right)\right)^{\alpha-1}\right], \alpha \neq 1, \alpha>0
$$

It has been proved that the maximum fuzzy cross entropy is a continuous and piecewise convex function of $k$. For illustration, a numerical example is used to show that the maximum cross entropy is monotonically decreasing when it is given that $\mu_{B}\left(x_{i}\right)$ is monotonically increasing for $1 \leq i \leq n$. Finally, a graph for maximum fuzzy cross entropy against the values of $0 \leq k \leq n$ is also drawn.

\section{Maximum Value of $D_{\alpha}(A: B)$ :}

Since $D_{\alpha}(A: B)$ is a measure of generalizes fuzzy entropy, its maximum value exits.Consider the following cases:

Case I : When $k$ is any positive integer, say $k=m$, then we can choose $m$ values of $\mu_{A}\left(x_{i}\right)$ as unity and remaining $(n-m)$ values as zero. i.e., $\mu_{A}\left(x_{i}\right)=(1,1,1, \ldots \ldots 1,0,0, \ldots \ldots . .0)$.In this case, From equation (1), we have

$D_{\alpha}(A: B)=\frac{1}{\alpha-1}\left[\sum_{i=1}^{m} \log \left[\mu_{A}^{\alpha}\left(x_{i}\right) \mu_{B}^{1-\alpha}\left(x_{i}\right)+\left(1-\mu_{A}\left(x_{i}\right)\right)^{\alpha}\left(1-\mu_{B}\left(x_{i}\right)\right)^{1-\alpha}\right]+\sum_{i=m+1}^{n} \log \left[\mu_{A}^{\alpha}\left(x_{i}\right) \mu_{B}^{1-\alpha}\left(x_{i}\right)+\left(1-\mu_{A}\left(x_{i}\right)\right)^{\alpha}\left(1-\mu_{B}\left(x_{i}\right)\right)^{1-\alpha}\right]\right]$

$\therefore \operatorname{Max} . D_{\alpha}(A: B)=-\left[\sum_{i=1}^{m} \log \mu_{B}\left(x_{i}\right)+\sum_{i=m+1}^{n} \log \left(1-\mu_{B}\left(x_{i}\right)\right)\right]$

\subsection{Illustration :}

Suppose $\mu_{B}\left(x_{i}\right)$ is monotonically decreasing i.e., $\mu_{B}\left(x_{i}\right)=\left(\frac{1}{2}, \frac{1}{3}, \frac{1}{4}, \ldots \ldots \frac{1}{n+1}\right)$

(i) When $m=0$, equation (3) gives

$$
\operatorname{Max} . D_{\alpha}(A: B)=-\sum_{i=1}^{n} \log \left(1-\mu_{B}\left(x_{i}\right)\right)=-\left(\log \frac{1}{2}+\log \frac{2}{3}+\log \frac{3}{4}+\ldots \ldots+\log \frac{n}{n+1}\right)=\log (n+1)
$$

(ii) When $m=1$, equation (3) gives

$$
\operatorname{Max.} D_{\alpha}(A: B)=-\left[\log \mu_{B}\left(x_{i}\right)+\sum_{i=2}^{n} \log \left(1-\mu_{B}\left(x_{i}\right)\right)\right]=\log (n+1)
$$

(iii) When $m=2$, equation (3) gives

$$
\operatorname{Max.} D_{\alpha}(A: B)=-\left[\log \mu_{B}\left(x_{1}\right)+\log \mu_{B}\left(x_{2}\right)+\sum_{i=3}^{n} \log \left(1-\mu_{B}\left(x_{i}\right)\right)\right]=\log 2+\log (n+1)
$$


(iv) When $m=3$, equation (3) gives

$$
\operatorname{Max} . D_{\alpha}(A: B)=-\left[\log \mu_{B}\left(x_{1}\right)+\log \mu_{B}\left(x_{2}\right)+\sum_{i=3}^{n} \log \left(1-\mu_{B}\left(x_{i}\right)\right)\right]=\log 2+\log 3+\log (n+1)
$$

(v) When $m=4$, equation (3) gives

$$
\operatorname{Max} . D_{\alpha}(A: B)=\log 2+\log 3+\log 4+\log (n+1)
$$

(vi) When $m=n$, equation (3) gives

$$
\operatorname{Max.} D_{\alpha}(A: B)=-\sum_{i=1}^{n} \log \mu_{B}\left(x_{i}\right)=-\left(\log \frac{1}{2}+\log \frac{1}{3}+\log \frac{1}{4}+\ldots \ldots+\log \frac{1}{n+1}\right)=\log \mid n+1
$$

The graph of $\operatorname{Max} . D_{\alpha}(A: B)$ against the values $0 \leq k \leq n$ is shown in the following figure 1 :

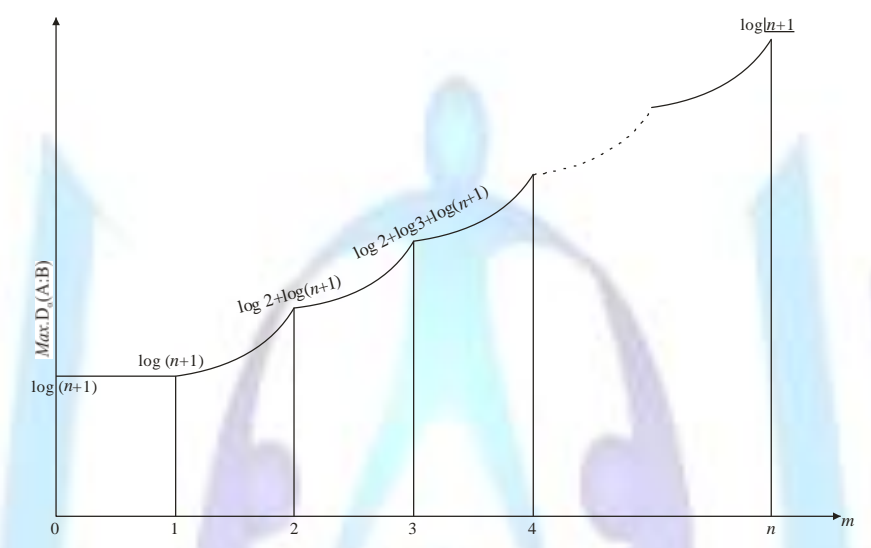

Figure 1: Maximum values of $D_{\alpha}(A: B)$ against the values $0 \leq k \leq n$

From figure 1 , we conclude that $\operatorname{Max} \cdot D_{\alpha}(A: B)$ is monotonically increasing when $\mu_{B}\left(x_{i}\right)$ is monotonically decreasing. Also, the $\operatorname{Max} \cdot D_{\alpha}(A: B)$ is a piecewise convex function of $k$.

Case II : When $k=m+\xi$, where $m$ is any non-negative integer and $\xi$ is a positive fraction. We put $m$ values of $\mu_{A}\left(x_{i}\right)$ as unity, $(m+1)^{\text {th }}$ values as $\xi$ and remaining $(n-m-1)$ values as zero i.e., $\mu_{A}\left(x_{i}\right)=(1,1,1, \ldots . .1, \xi, 0,0, \ldots 0)$.

In this case, Using equation (1), we have

$$
\begin{aligned}
& D_{\alpha}(A: B)=\frac{1}{\alpha-1}\left[\begin{array}{r}
\sum_{i=1}^{m} \log \left[\mu_{A}^{\alpha}\left(x_{i}\right) \mu_{B}^{1-\alpha}\left(x_{i}\right)+\left(1-\mu_{A}\left(x_{i}\right)\right)^{\alpha}\left(1-\mu_{B}\left(x_{i}\right)\right)^{1-\alpha}\right]+\log \left[\mu_{A}^{\alpha}\left(x_{m+1}\right) \mu_{B}^{1-\alpha}\left(x_{m+1}\right)+\left(1-\mu_{A}\left(x_{m+1}\right)\right)^{\alpha}\left(1-\mu_{B}\left(x_{m+1}\right)\right)^{1-\alpha}\right] \\
+\sum_{i=m+2}^{n} \log \left[\mu_{A}^{\alpha}\left(x_{i}\right) \mu_{B}^{1-\alpha}\left(x_{i}\right)+\left(1-\mu_{A}\left(x_{i}\right)\right)^{\alpha}\left(1-\mu_{B}\left(x_{i}\right)\right)^{1-\alpha}\right]
\end{array}\right] \\
& \therefore \quad \operatorname{Max} . D_{\alpha}(A: B)=-\sum_{i=1}^{m} \log \mu_{B}\left(x_{i}\right)+\frac{1}{\alpha-1} \log \left[\xi^{\alpha} \mu_{B}^{1-\alpha}\left(x_{m+1}\right)+(1-\xi)^{\alpha}\left(1-\mu_{B}\left(x_{m+1}\right)\right)^{1-\alpha}\right]-\sum_{i=m+2}^{n} \log \left(1-\mu_{B}\left(x_{i}\right)\right)
\end{aligned}
$$

\subsection{Illustration :}

Suppose $\mu_{B}\left(x_{i}\right)$ is monotonically decreasing, so we take

$\mu_{B}\left(x_{i}\right)=\left(\frac{1}{2}, \frac{1}{3}, \frac{1}{4}, \ldots . . \frac{1}{m+1}, \frac{1}{m+2}, \frac{1}{m+3}, \frac{1}{m+4}, \ldots \ldots \frac{1}{n+1}\right)$

Using, equation (1), we have

Max. $D_{\alpha}(A: B)=\log 2+\log 3+\log 4+\ldots . .+\log (m+1)+\log \frac{m+3}{m+2}+\log \frac{m+4}{m+3}+\ldots \ldots+\log \frac{n+1}{n}+\frac{1}{\alpha-1} \log \left[\xi^{\alpha}(m+2)^{\alpha-1}+(1-\xi)^{\alpha}\left(\frac{m+2}{m+1}\right)^{\alpha-1}\right]$

$=\log (n+1)\lfloor m+\phi(\xi)$, where

$$
\phi(\xi)=\frac{1}{\alpha-1} \log \left(\xi^{\alpha}(m+1)^{\alpha-1}+(1-\xi)^{\alpha}\right)
$$




\subsection{Convexity of $\phi(\xi)$ :}

Take $h(\xi)=\xi^{\alpha}(m+1)^{\alpha-1}+(1-\xi)^{\alpha}$. Differentiating it w.r.t. $\xi$, we get

$h^{\prime}(\xi)=\alpha\left(\xi^{\alpha-1}(m+1)^{\alpha-1}-(1-\xi)^{\alpha-1}\right) ; h^{\prime \prime}(\xi)=\left(\alpha^{2}-\alpha\right)\left(\xi^{\alpha-2}(m+1)^{\alpha-1}+(1-\xi)^{\alpha-2}\right)$

(i) If $\alpha>1$, then $h^{\prime \prime}(\xi)>0 \Rightarrow h(\xi)$ is a convex function of $\xi \Rightarrow \log h(\xi)$ is a convex function of $\xi \Rightarrow \frac{1}{\alpha-1} \log h(\xi)$ is a convex function of $\xi$ for each $\alpha>1$.

(ii) If $0<\alpha<1$, then $h^{\prime \prime}(\xi)<0 \Rightarrow h(\xi)$ is a concave function of $\xi \Rightarrow \log h(\xi)$ is a concave function of $\xi \Rightarrow \frac{1}{\alpha-1} \log h(\xi)$ is a convex function of $\xi$ for each $0<\alpha<1$

Thus, $\phi(\xi)$ is a convex function of $\xi$ for each $\alpha$. So, its minimum value exists. For minimum, we put

$\frac{d}{d \xi} \phi(\xi)=0$, which gives $\frac{\alpha}{\alpha-1} \frac{\xi^{\alpha-1}(m+1)^{\alpha-1}-(1-\xi)^{\alpha-1}}{\xi^{\alpha}(m+1)^{\alpha-1}+(1-\xi)^{\alpha}}=0$

| Using equation (6)

$\Rightarrow \quad \xi(m+2)-1=0 \quad \Rightarrow \quad \xi=\frac{1}{m+2}$

Therefore, the minimum value of $\phi(\xi)$ exists at $\xi=\frac{1}{m+2}$. Hence, from equation (8), the minimum value of $\operatorname{Max} . D_{\alpha}(A: B)$ is :

$\operatorname{Min} \cdot \operatorname{Max} . D_{\alpha}(A: B)=\log \underline{m}(n+1)+\phi\left(\frac{1}{m+2}\right)=\log \underline{m}(n+1)+\frac{1}{\alpha-1} \log \left(\frac{(m+1)^{\alpha-1}}{(m+2)^{\alpha}}+\left(\frac{m+1}{m+2}\right)^{\alpha}\right)$

$$
=\log \frac{(n+1)\lfloor\underline{m}(m+1)}{m+2}=\log \frac{(n+1)\lfloor m+1}{m+2}=\log (n+1)+\log \frac{\mid m+1}{m+2}
$$

For different values of $m$, the different values of $\operatorname{Min} \cdot \operatorname{Max} \cdot D_{\alpha}(A: B)$ is shown in the following table 1

\begin{tabular}{|c|c|}
\hline Value of $m$ & $\begin{array}{c}\text { Minimum value of } \\
\text { Min.Max. } D_{\alpha}(A: B)\end{array}$ \\
\hline$m=0$ & $\log \frac{(n+1) \underline{\underline{1}}}{2}$ \\
\hline$m=1$ & $\log \frac{(n+1)\lfloor\underline{2}}{3}$ \\
\hline$m=2$ & $\log \frac{(n+1) \underline{3}}{4}$ \\
\hline$m=3$ & $\log \frac{(n+1)\lfloor\underline{4}}{5}$ \\
\hline$m=n-1$ & $\log \frac{(n+1) \underline{n}}{n+1}$ \\
\hline$m=n$ & $\log \frac{(n+1)\lfloor n+1}{n+2}$ \\
\hline
\end{tabular}

Table 1 : Minimum values of $\operatorname{Max} \cdot D_{\alpha}(A: B)$ against the values $0 \leq m \leq n$.

From table1, we can plot the graph of $\operatorname{Min} \cdot \operatorname{Max} \cdot D_{\alpha}(A: B)$ against the values of $0 \leq m \leq n$ as shown in the following figure 2 


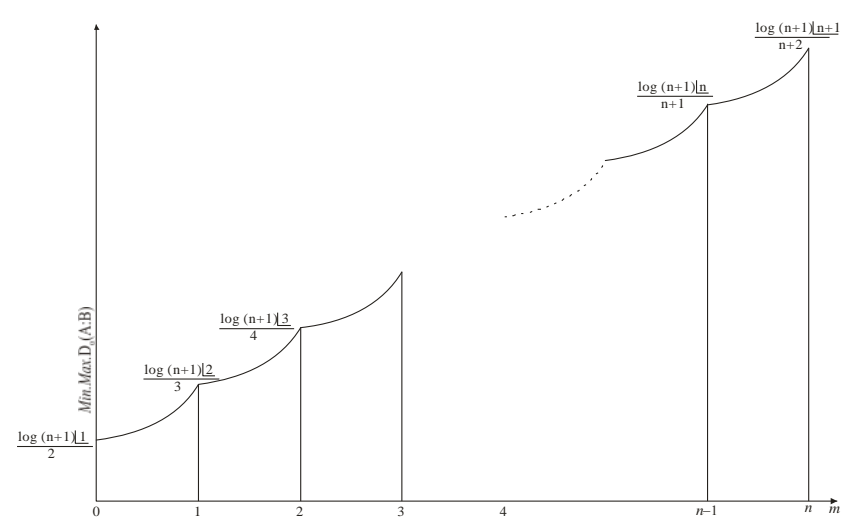

Figure : 2 Minimum value of $\operatorname{Max} . D_{\alpha,}(A: B)$ against the values $0 \leq m \leq n$

From figure 2, we conclude that when $\mu_{B}\left(x_{i}\right)$ is monotonically decreasing, $\operatorname{Max} \cdot D_{\alpha}(A: B)$ is monotonically increasing. Also Min.Max. $D^{\alpha}(A: B)$ is piecewise convex function of $k$.

\section{Maximum Value of $D^{\alpha}(A: B)$ :}

Consider the following cases:

Case $\mathbf{I}$ : When $k$ is any positive integer, say $k=m$, then, we can choose $m$ values of $\mu_{A}\left(x_{i}\right)$ as unity and remaining $n-m$ values as zero i.e., $\mu_{A}\left(x_{i}\right)=(1,1,1, \ldots . .1,0,0, \ldots \ldots .0)$

In this case,from equation (2), we have

$$
\begin{gathered}
D^{\alpha}(A: B)=\frac{1}{\alpha-1}\left[\sum_{i=1}^{m} \mu_{A}^{\alpha}\left(x_{i}\right) \mu_{B}^{1-\alpha}\left(x_{i}\right)+\left(1-\mu_{A}\left(x_{i}\right)\right)^{\alpha}\left(1-\mu_{B}\left(x_{i}\right)\right)^{1-\alpha}-1+\sum_{i=m+1}^{n}\left[\begin{array}{l}
\mu_{A}^{\alpha}\left(x_{i}\right) \mu_{B}^{1-\alpha}\left(x_{i}\right) \\
+\left(1-\mu_{A}\left(x_{i}\right)\right)^{\alpha}\left(1-\mu_{B}\left(x_{i}\right)\right)^{1-\alpha}-1
\end{array}\right]\right. \\
\therefore \operatorname{Max}^{\alpha}(A: B)=\frac{1}{\alpha-1}\left[\sum_{i=1}^{m}\left(\mu_{B}^{1-\alpha}\left(x_{i}\right)-1\right)+\sum_{i=m+1}^{n}\left(\left(1-\mu_{B}\left(x_{i}\right)\right)^{1-\alpha}-1\right)\right]
\end{gathered}
$$

\subsection{Illustration :}

Suppose $\mu_{B}\left(x_{i}\right)$ is monotonically decreasing i.e., $\mu_{B}\left(x_{i}\right)=\left(\frac{1}{2}, \frac{1}{3}, \frac{1}{4}, \ldots \ldots \frac{1}{n+1}\right)$

(i) When $m=0$, equation (7) gives

$$
\operatorname{Max} . D^{\alpha}(A: B)=\frac{1}{\alpha-1}\left[\sum_{i=1}^{n}\left[\left(1-\mu_{B}\left(x_{i}\right)\right)^{1-\alpha}-1\right]\right]=\frac{1}{\alpha-1}\left[2^{\alpha-1}+\left(\frac{3}{2}\right)^{\alpha-1}+\left(\frac{4}{3}\right)^{\alpha-1}+\ldots \ldots+\left(\frac{n+1}{n}\right)^{\alpha-1}-n\right]
$$

(ii) When $m=1$, equation (7) gives

$$
\operatorname{Max} . D^{\alpha}(A: B)=\frac{1}{\alpha-1}\left[\left(\mu_{B}^{1-\alpha}\left(x_{i}\right)-1\right)+\sum_{i=2}^{n}\left[\left(1-\mu_{B}\left(x_{i}\right)\right)^{1-\alpha}-1\right]\right]=\frac{1}{\alpha-1}\left[2^{\alpha-1}+\left(\frac{3}{2}\right)^{\alpha-1}+\left(\frac{4}{3}\right)^{\alpha-1}+\ldots \ldots+\left(\frac{n+1}{n}\right)^{\alpha-1}-n\right]
$$

(iii) When $m=2$, equation (7) gives

$$
\begin{aligned}
\operatorname{Max} . D^{\alpha}(A: B) & =\frac{1}{\alpha-1}\left\{\left(\mu_{B}^{1-\alpha}\left(x_{i}\right)-1\right)+\left(\mu_{B}^{1-\alpha}\left(x_{2}\right)-1\right)+\sum_{i=3}^{n}\left[\left(1-\mu_{B}\left(x_{i}\right)\right)^{1-\alpha}-1\right]\right\} \\
& =\frac{1}{\alpha-1}\left[2^{\alpha-1}+3^{\alpha-1}+\left(\frac{4}{3}\right)^{\alpha-1}+\left(\frac{5}{4}\right)^{\alpha-1}+\ldots \ldots+\left(\frac{n+1}{n}\right)^{\alpha-1}-n\right]
\end{aligned}
$$

(iv) When $m=3$, equation (7) gives

$$
\operatorname{Max} . D^{\alpha}(A: B)=\frac{1}{\alpha-1}\left[2^{\alpha-1}+3^{\alpha-1}+4^{\alpha-1}+\left(\frac{5}{4}\right)^{\alpha-1}+\left(\frac{6}{5}\right)^{\alpha-1}+\ldots \ldots+\left(\left(\frac{n+1}{n}\right)^{\alpha-1}-n\right)\right]
$$




$$
\operatorname{Max} . D^{\alpha}(A: B)=\frac{1}{\alpha-1}\left[2^{\alpha-1}+3^{\alpha-1}+4^{\alpha-1}+\ldots \ldots+(n+1)^{\alpha-1}-n\right]
$$

The graph of $\operatorname{Max} . D^{\alpha}(A: B)$ against the values $0 \leq k \leq n$ is shown in the following figure 3:

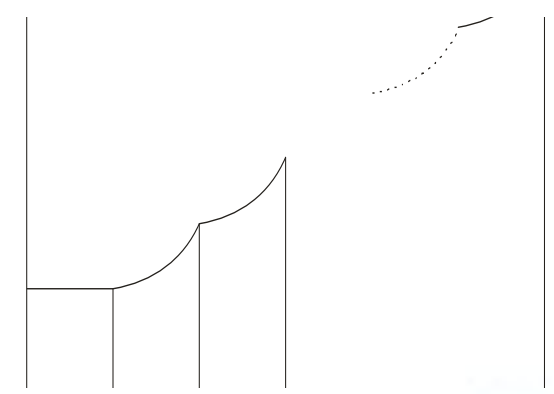

Figure 3: Maximum value of $D^{\alpha}(A: B)$ against the values $0 \leq k \leq n$

From figure 3, we conclude that $\operatorname{Max} \cdot D^{\alpha}(A: B)$ is monotonically increasing when $\mu_{B}\left(x_{i}\right)$ is monotonically decreasing. Also, the $\operatorname{Max} . D^{\alpha}(A: B)$ is a piecewise convex function of $k$.

Case II. When $k=m+\xi$, where $m$ is any non-negative integer and $\xi$ is a positive fraction. We put $m$ values of $\mu_{A}\left(x_{i}\right)$ as unity, $(m+1)^{\text {th }}$ value as $\xi$ and remaining $(n-m-1)$ values as zero i.e., $\mu_{A}\left(x_{i}\right)=(1,1,1, \ldots \ldots 1, \xi, 0,0, \ldots \ldots 0)$. Using equation (2), we have

$$
\begin{aligned}
& D^{\alpha}(A: B)=\frac{1}{\alpha-1}\left[\begin{array}{c}
\sum_{i=1}^{m}\left[\mu_{A}^{\alpha}\left(x_{i}\right) \mu_{B}^{1-\alpha}\left(x_{i}\right)+\left(1-\mu_{A}\left(x_{i}\right)\right)^{\alpha}\left(1-\mu_{B}\left(x_{i}\right)\right)^{1-\alpha}-1\right]+\mu_{A}^{\alpha}\left(x_{m+1}\right) \mu_{B}^{1-\alpha}\left(x_{m+1}\right)+\left(1-\mu_{A}\left(x_{m+1}\right)\right)^{\alpha}\left(1-\mu_{B}\left(x_{m+1}\right)\right)^{1-\alpha}-1 \\
+\sum_{i=m+2}^{n}\left[\mu_{A}^{\alpha}\left(x_{i}\right) \mu_{B}^{1-\alpha}\left(x_{i}\right)+\left(1-\mu_{A}\left(x_{i}\right)\right)^{\alpha}\left(1-\mu_{B}\left(x_{i}\right)\right)^{1-\alpha}-1\right]
\end{array}\right] \therefore \\
& \operatorname{Max} . D^{\alpha}(A: B)=\frac{1}{\alpha-1}\left[\sum_{i=1}^{m}\left(\mu_{B}^{1-\alpha}\left(x_{i}\right)-1\right)+\xi^{\alpha} \mu_{B}^{1-\alpha}\left(x_{m+1}\right)+(1-\xi)^{\alpha}\left(1-\mu_{B}\left(x_{m+1}\right)\right)^{1-\alpha}-1+\sum_{i=m+2}^{n}\left[\left(1-\mu_{B}\left(x_{i}\right)\right)^{1-\alpha}-1\right]\right]
\end{aligned}
$$

3.2 Illustration :

Suppose $\mu_{B}\left(x_{i}\right)$ is monotonically decreasing i.e.; $\mu_{B}\left(x_{i}\right)=\left(\frac{1}{2}, \frac{1}{3}, \frac{1}{4}, \ldots \ldots \frac{1}{m+1}, \frac{1}{m+2}, \frac{1}{m+3}, \ldots \ldots . \frac{1}{n+1}\right)$, from equation (2), we have

$$
\begin{aligned}
\operatorname{Max} . D^{\alpha}(A: B)=\frac{1}{\alpha-1} & \left(2^{\alpha-1}+3^{\alpha-1}+\ldots \ldots+(m+1)^{\alpha-1}\right)+\frac{1}{\alpha-1}\left[\left(\frac{m+3}{m+2}\right)^{\alpha-1}+\left(\frac{m+4}{m+3}\right)^{\alpha-1}+\ldots \ldots+\left(\frac{n+1}{n}\right)^{\alpha-1}-n\right] \\
& +\phi(\xi), \text { where } \phi(\xi)=\frac{1}{\alpha-1}\left(\xi^{\alpha}(m+2)^{\alpha-1}+(1-\xi)^{\alpha}\left(\frac{m+2}{m+1}\right)^{\alpha-1}\right)
\end{aligned}
$$

\subsection{Convexity of $\phi(\xi)$ :}

It can be easily proved that the function $\phi(\xi)$ is a convex function of $\xi$ for each value of $\alpha$. Hence the minimum value exits and it exits at $\xi=\frac{1}{m+2}$. Further,

$$
\operatorname{Min} . \operatorname{Max} . D^{\alpha}(A: B)=\frac{1}{\alpha-1}\left[2^{\alpha-1}+3^{\alpha-1}+4^{\alpha-1}+\ldots \ldots+(m+1)^{\alpha-1}+\left(\frac{m+3}{m+2}\right)^{\alpha-1}+\left(\frac{m+4}{m+3}\right)^{\alpha-1}+\ldots \ldots+\left(\frac{n+1}{n}\right)^{\alpha-1}-n\right]+\frac{1}{\alpha-1}
$$

(i) When $m=0$, equation (9) gives

$$
\operatorname{Min} \cdot \operatorname{Max} . D^{\alpha}(A: B)=\frac{1}{\alpha-1}\left[1+\left(\frac{3}{2}\right)^{\alpha-1}+\left(\frac{4}{3}\right)^{\alpha-1}+\ldots \ldots+\left(\frac{n+1}{n}\right)^{\alpha-1}-n+1\right]=g_{0}(\alpha) \text {, say }
$$

(ii) When $m=1$, equation (9) gives

$$
\operatorname{Min} \cdot \operatorname{Max} . D^{\alpha}(A: B)=\frac{1}{\alpha-1}\left[2^{\alpha-1}+\left(\frac{4}{3}\right)^{\alpha-1}+\left(\frac{5}{4}\right)^{\alpha-1}+\ldots \ldots+\left(\frac{n+1}{n}\right)^{\alpha-1}-n+1\right]=g_{1}(\alpha) \text {, say }
$$


(iii) When $m=2$, equation (9) gives

$$
\operatorname{Min} . \operatorname{Max} . D^{\alpha}(A: B)=\frac{1}{\alpha-1}\left[2^{\alpha-1}+3^{\alpha-1}+\left(\frac{5}{4}\right)^{\alpha-1}+\left(\frac{6}{5}\right)^{\alpha-1}+\ldots \ldots+\left(\frac{n+1}{n}\right)^{\alpha-1}-n+1\right]=g_{2}(\alpha) \text {, say }
$$

(iv) When $m=3$, equation (9) gives

$$
\operatorname{Min} . \operatorname{Max} . D^{\alpha}(A: B)=\frac{1}{\alpha-1}\left[2^{\alpha-1}+3^{\alpha-1}+4^{\alpha-1}+\left(\frac{6}{5}\right)^{\alpha-1}+\left(\frac{7}{6}\right)^{\alpha-1}+\ldots \ldots+\left(\frac{n+1}{n}\right)^{\alpha-1}-n+1\right]=g_{3}(\alpha) \text {, say }
$$

(v) When $m=n$, equation (9) gives

$$
\operatorname{Min} . \operatorname{Max} . D^{\alpha}(A: B)=\frac{1}{\alpha-1}\left[2^{\alpha-1}+3^{\alpha-1}+4^{\alpha-1}+\ldots \ldots+(n+1)^{\alpha-1}-n+1\right]=g_{n}(\alpha) \text {, say }
$$

The graph of Min.Max. $D^{\alpha}(A: B)$ against the values of $0 \leq m \leq n$ is shown in the following figure 4:

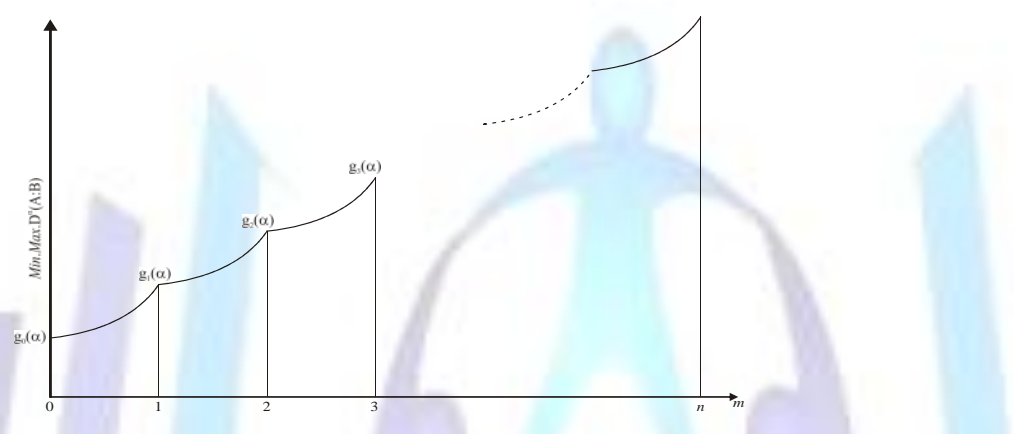

Figure 4 : Minimum value of $\operatorname{Max} . D^{\alpha}(A: B)$ against the values $0 \leq k \leq n$

From figure 4, we conclude that $\operatorname{Max}^{\alpha}(A: B)$ is monotonically increasing when $\mu_{B}\left(x_{i}\right)$ is monotonically decreasing. Also $\operatorname{Max} . D^{\alpha}(A: B)$ is a piecewise convex function of $k$.

Conclusion : It has been proved that the maximum generalized fuzzy cross entropy is a continuous and piecewise convex function of $k$. Also, the maximum generalized fuzzy cross entropy is monotonically decreasing when it is given that $\mu_{B}\left(x_{i}\right)$ is monotonically increasing.,

Acknowledgement: The authors are thankful to Bahra University, Shimla, India for providing the technical support in the preparation of this manuscript.

Funding Acknowledgement : This research paper received no specific grant from any funding agency either publically, commercially or private sectors.

\section{REFERENCES}

[1] Aczel, J. and Daroczy, Z. (1975). On measures of information and their characterizations. Academic Press 115: 219226.

[2] Havrada, J.H. and Charvat, F. (1967). Quantification methods of classification process:Concept of structural $\alpha$ entropy. Kybernetika 3: 30-35.

[3] Kapur, J.N. (1997). Measures of Fuzzy Information. Mathematical Sciences Trust Society, New Delhi.

[4] Renyi, A. (1961). On measures of entropy and information. Proceedings 4th Berkeley Symposium on Mathematical Statistics and Probability 1: 547-561.

[5] Zadeh, L. A. (1965). Fuzzy sets. Information and Control 8 : 338-353. 